\title{
A comparison of newer classifications of bronchopulmonary dysplasia: findings from the Children's Hospitals Neonatal Consortium Severe BPD Group
}

\author{
Shilpa Vyas-Read (iD ${ }^{1 凶}$, J. Wells Logan (D) ${ }^{2}$, Alain C. Cuna $\left(D^{3}{ }^{3}\right.$, Joana Machry ${ }^{4}$, Kristin T. Leeman (iD ${ }^{5}$, Rebecca S. Rose (iD ${ }^{6}$, \\ Michel Mikhael (iD ${ }^{7}$, Erica Wymore ${ }^{8}$, John W. Ibrahim ${ }^{9}$, Robert J. DiGeronimo (D) ${ }^{10}$, Sushmita Yallapragada (iD ${ }^{11}$, Beth E. Haberman ${ }^{12}$, \\ Michael A. Padula (iD ${ }^{13}$, Nicolas F. Porta (iD ${ }^{14}$, Karna Murthy ${ }^{14}$, Leif D. Nelin ${ }^{2}$, Carl H. Coghill (iD) ${ }^{15}$, Isabella Zaniletti (D) ${ }^{16}$, \\ Rashmin C. Savani $\mathbb{D}^{11}$, William Truog ${ }^{3}$, William A. Engle $\mathbb{D}^{6}$ and Joanne M. Lagatta (D) $^{17}$
}

(c) The Author(s), under exclusive licence to Springer Nature America, Inc. 2021

\begin{abstract}
OBJECTIVE: To compare three bronchopulmonary dysplasia (BPD) definitions against hospital outcomes in a referral-based population.

STUDY DESIGN: Data from the Children's Hospitals Neonatal Consortium were classified by 2018 NICHD, 2019 NRN, and Canadian Neonatal Network (CNN) BPD definitions. Multivariable models evaluated the associations between BPD severity and death, tracheostomy, or length of stay, relative to No BPD references.

RESULTS: Mortality was highest in 2019 NRN Grade 3 infants (aOR 225), followed by 2018 NICHD Grade 3 (aOR 145). Infants with lower BPD grades rarely died $(<1 \%)$, but Grade 2 infants had aOR 7-21-fold higher for death and 23-56-fold higher for tracheostomy.

CONCLUSIONS: Definitions with 3 BPD grades had better discrimination and Grade 32019 NRN had the strongest association with outcomes. No/Grade 1 infants rarely had severe outcomes, but Grade 2 infants were at risk. These data may be useful for counseling families and determining therapies for infants with BPD.
\end{abstract}

Journal of Perinatology (2022) 42:58-64; https://doi.org/10.1038/s41372-021-01178-4

\section{INTRODUCTION}

Bronchopulmonary dysplasia (BPD) has been described by a changing set of definitions since its first description by Northway in 1967 [1]. Shennan and colleagues introduced the idea that infants who required respiratory support at 36 weeks' postmenstrual age (PMA) were more likely to have abnormal pulmonary outcomes in the first 2 years of life [2]. Later, to capture the changing BPD phenotypes seen after the advent of surfactant and antenatal steroids, a severity-based classification was proposed in a 2001 National Institute of Child Health and Human Development (NICHD) Workshop [3, 4]. Challenges emerged in applying the 2001 NICHD BPD definition as newer therapies, such as high-flow nasal cannula and non-invasive mechanical ventilation, sometimes without supplemental oxygen, were more widely used [5-8].

To address classification dilemmas, new BPD definitions have been proposed [9-11]. Each proposed definition of BPD has added to the granularity of classification, ease of use, and identification of infants at the highest risk for poor outcomes. However, differences in the criteria utilized, and variations in outcome measures have created uncertainty in the clinical, epidemiologic, and research applications of the classifications. The Children's Hospitals Neonatal Consortium (CHNC) includes 34 neonatal intensive care units (NICUs) of largely outborn infants, who are referred into quaternary care centers for surgical and sub-specialty care [12]. As many infants with severe BPD are ultimately referred to CHNC centers for evaluation and treatment of pulmonary and nonpulmonary co-morbid conditions, it is essential that adopted BPD definitions are generalizable to this population. Further, an understanding of how hospital-based outcomes differ by definition within this high-acuity referral population is fundamental to defining the populations at risk for adverse events.

In this study, we performed a comparison of three recent BPD definitions using a common reference group and evaluated the

\footnotetext{
${ }^{1}$ Emory University and Children's Healthcare of Atlanta, Atlanta, GA, USA. ${ }^{2}$ The Ohio State University and Nationwide Children's Hospital, Columbus, OH, USA. ${ }^{3}$ University of

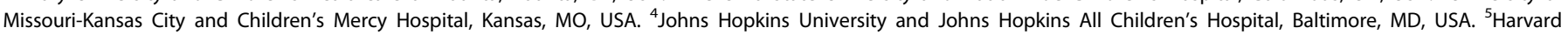

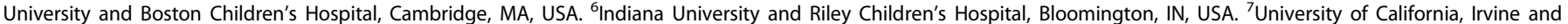

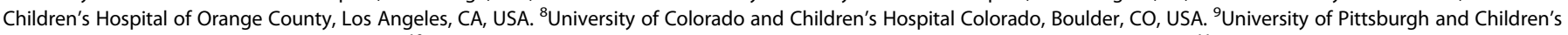

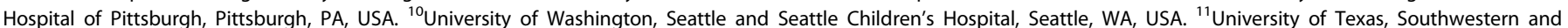

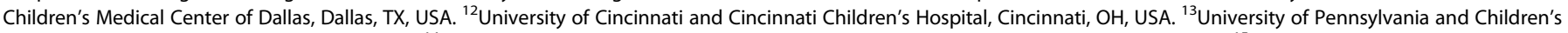

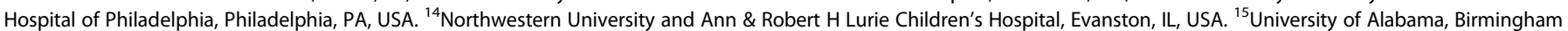

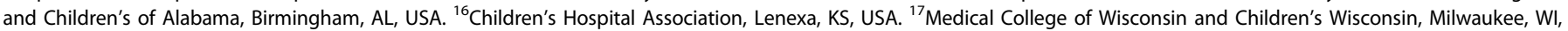
USA. ${ }^{凶}$ email: svyasre@emory.edu
} 
performance of each definition on the outcomes of death and hospital-based morbidity in a high-acuity, referral-based neonatal population.

\section{METHODS}

We performed a retrospective analysis of data from the CHNC from January 2010 to December 2016. Infant clinical data were collected by trained chart abstractors at each site, with periodic assessments of inter-rater agreement scores. De-identified data analyses were approved by the Institutional Review Board of Stanley Manne Research Institute affiliated with the Ann and Robert H. Lurie Children's Hospital of Chicago. All participating centers obtained regulatory oversight to participate in the CHNC [12].

Infants born $<32$ weeks' gestational age who were admitted to a CHNC neonatal intensive care unit at $<14$ days of life were eligible for inclusion in the study cohort. Infants were excluded if they had a length of stay (LOS) of $<3$ days; died before or on day of life 14; had significant congenital anomalies; or were re-admitted following hospital discharge, transferred to another facility prior to hospital discharge, or not discharged from the hospital at 1 year of life.

The primary outcome for the study was death between 36 weeks' PMA and hospital discharge, and secondary outcomes included LOS or the need for supportive therapies, such as tracheostomy, at the time of discharge. Data on PMA in weeks at the time of death or discharge, therapy with nasogastric or surgically-placed feeding modalities, and pulmonary medications were also collected and compared within definitions.

\section{BPD definitions}

Infants were classified into one of three BPD definitions based on the criteria laid out in prior publications [9-11]. For the 2018 NICHD definition, infants were categorized into "No BPD" or Grade 1-3 BPD as previously published [9]. For the 2019 NRN definition, infants were categorized as having "No BPD" or grades 1-3 BPD as previously published [10]. In the original CNN publication, infants were categorized as having BPD if they required respiratory support (>1.5 LPM NC, NCPAP, NIMV, and mechanical ventilation) and oxygen between 34 and 44 weeks' PMA, and the infants who were on this level of respiratory support at 36 weeks' PMA were categorized "CNN BPD" in our cohort. In addition, to retain a common reference group of infants receiving no respiratory support at 36 weeks, alongside the CNN definition we created an additional group for those infants on respiratory support $\leq 1.5$ LPM NC labeled "CNN RS" [11]. A full description of the definitions used for classification is available in Supplementary Table 1.

\section{Demographic and hospital characteristics}

We evaluated baseline maternal demographic variables including maternal race, diabetes, chorioamnionitis, hypertension, the multiplicity of birth, receipt of antenatal steroids, and mode of delivery. Baseline neonatal variables included the gestational age (in weeks), percentage of inborn infants and the PMA at admission, birthweight, sex, and percentage of growth-restricted infants (weight $<10 \%$ for gestational age). Growth restriction was defined using previously published growth charts for gestational-age-specific data [13]. Respiratory variables included ventilator days, postnatal systemic steroids, airway co-morbidities, and the diagnosis of pulmonary hypertension in the medical record. Postnatal steroids were defined as the receipt of IV or oral steroids, including hydrocortisone or dexamethasone, but did not include inhaled corticosteroids. Other neonatal co-morbidities were defined as follows: patent ductus arteriosus ligation, ductus arteriosus with left-to-right or bidirectional shunting not associated with congenital heart disease; necrotizing enterocolitis, surgical evidence or necrotizing enterocolitis or clinical symptoms (bilious emesis, abdominal distention, hematochezia) with radiographic findings (pneumatosis intestinalis, pneumoperitoneum, hepatobiliary gas); bloodstream infections, laboratory-confirmed bacterial or fungal infection; severe intraventricular hemorrhage, radiographic report of grade 3 or 4 intraventricular hemorrhage; and periventricular leukomalacia, cystic periventricular leukomalacia diagnosis on cranial US, CT, or MRI.

\section{Statistical analyses}

First, we compared baseline demographic and neonatal variables between severity levels within each of the 3 BPD definitions, using Chi-square and Fisher's exact tests for differences in proportions, and Kruskal-Wallis tests for continuous variables. Because the "No BPD" 2019 NRN, and the "none/ mild" CNN groups each had the same definition and were the same group of infants, these infants were used in further bivariate and multivariable analyses as a "common reference" group.

For each severity definition, we developed adjusted logistic regression models for the outcomes of death and tracheostomy. LOS was evaluated with a negative binomial regression. Multivariable models included adjustments for the center, gestational age, and gender with the median value for the center, and female gender serving as the reference values. Additional analyses evaluating the relative risks between different levels of respiratory support and $\mathrm{FiO}_{2}$ categories were performed. Statistical significance was defined as $P$ value $<0.05$. SAS Enterprise Guide v7.1N (SAS Institute, Cary, North Carolina) was used for analyses.

\section{RESULTS}

From 2010 to 2016, 4874 infants met inclusion criteria, 236 infants died between 14 days and 36 weeks' PMA, and 477 infants met exclusion criteria or had implausible outcome data, leaving 4161 infants in the final study cohort. These 4161 infants were then classified into the 3 BPD definitions, with 3-4 levels of severity within each definition (Fig. 1). The respiratory support and $\mathrm{FiO}_{2}$ criteria used to classify infants by definition are shown in Supplementary Table 1. The 2019 NRN and the CNN definitions had the same 1991 infants who did not meet the criteria for BPD. These infants were similar in hospital and demographic characteristics and were labeled as a "common reference group" for later logistic regression analyses. The "no BPD" group within the 2018 NICHD definition included 292 in excess of the other 2 definitions. More infants had Grade 1 BPD by the 2019 NRN classification (28\%) than by the 2018 NICHD definition (10\%), and most babies who had BPD by the 2019 NRN definition had Grade 1. Inversely, more babies had Grade 2 BPD by the 2018 NICHD definition (22\%), than by the 2019 NRN definition (16\%), and the largest number of babies with BPD by the 2018 NICHD definition had Grade 2. The smallest numbers of babies were classified as having Grade 3 BPD, for both the 2018 NICHD (13\%) and the 2019 NRN definitions (8\%). Thirty-one percent of the cohort had BPD by the CNN definition, and $21 \%$ were on $<1.5$ LPM of nasal cannula support but did not meet the criteria for BPD (labeled CNN RS).

The distribution of respiratory support and $\mathrm{FiO}_{2}$ for infants requiring support at 36 weeks' PMA in the study cohort is shown in Fig. 2. Overall, 2170 infants (52\%) were on some form of respiratory support at 36 weeks' PMA. Of those, the majority required $\leq 2$ LPM of NC flow (51\%), 31\% were on $>2$ LPM NC, NIMV, or NCPAP, and $\sim 16 \%$ were on invasive mechanical ventilation. The level of respiratory support and oxygen was then compared within each of the three definitions (Supplementary Fig. 1). Infants in the 2019 NRN grade 1 and CNN RS were similar and had respiratory support $\leq 1.5 \mathrm{LPM} \mathrm{NC}$ and $\mathrm{FiO}_{2}$ ranging $21-100 \%$. In contrast, the 2018 NICHD classification did not include infants who were on up to $3 \mathrm{LPM} \mathrm{NC}$ and $\mathrm{FiO}_{2} 21 \%$ within their BPD definition. Most infants who had BPD by the CNN definition had grade 2-3 BPD by the 2019 NRN definition. The major difference between those and the 2018 NICHD definition was that infants within the same respiratory support level (NC, NCPAP, NIMV) could be classified as grade 1,2 , or 3 BPD based on the level of $\mathrm{FiO}_{2}$.

\section{Short-term outcomes}

Rates of in-hospital mortality were approximately $0.1 \%$ for the lowest grades of BPD but increased significantly with BPD severity within each definition (Table 1). For CNN RS and Grade 1 BPD, death rates were $0.4-0.5 \%$, with the exception that infants classified as Grade 1 by the 2018 NICHD system had a mortality rate of $1.7 \%$. In-hospital death increased significantly for infants categorized as severe within each classification system. Infants categorized as severe by the 2019 NRN, 2018 NICHD, and CNN classifications system died $\sim 13 \%, 9 \%$, and $4 \%$ of the time, respectively. The discharge PMA also increased with increasing BPD severity within each classification, with infants with milder 


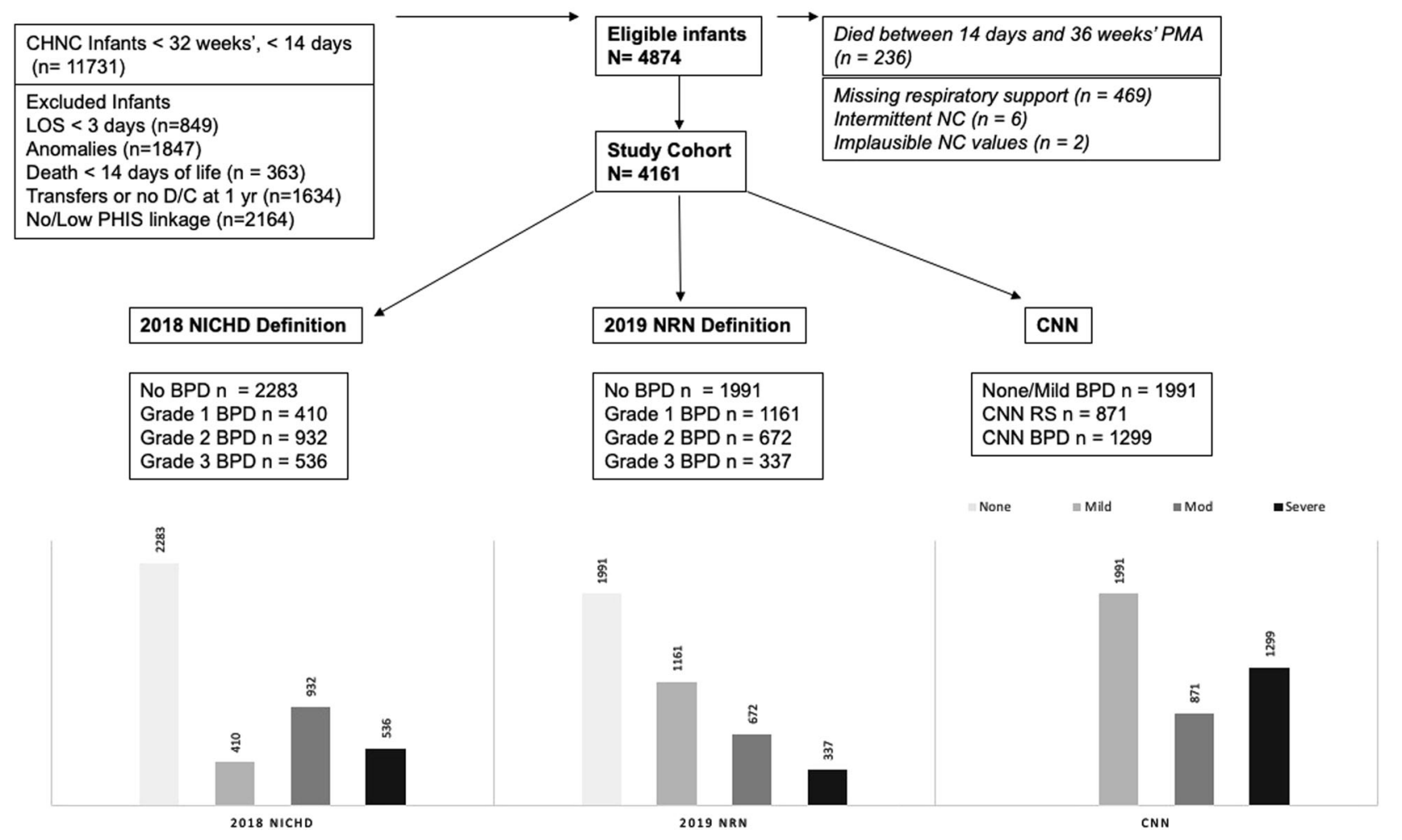

Fig. 1 Flow diagram for patient selection. Of eligible infants between 2010 and 2016, 4161 infants survived to BPD classification and had levels of respiratory support and oxygen recorded within the CHNC. Infants were classified into grades of BPD severity, based on the criteria outlined in 3 BPD definitions: the 2018 NICHD et al definition, the 2019 NRN et al definition, or the Canadian Neonatal Network Definition (CNN).

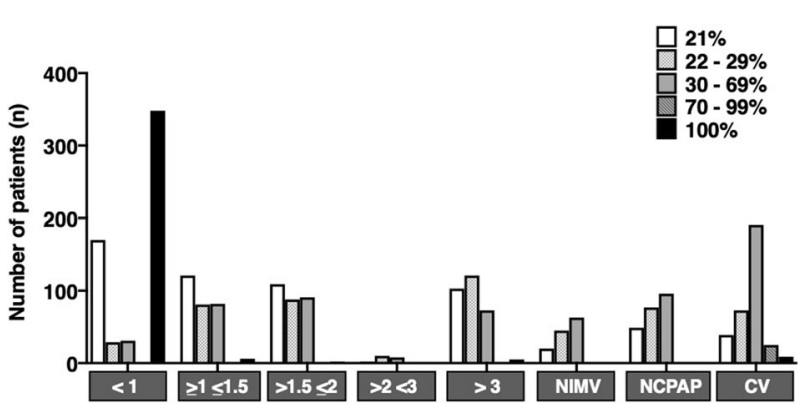

Fig. 2 Distribution of respiratory support and $\mathrm{FiO}_{2}$ among infants on support at 36 weeks' PMA. The bar graphs show the number of patients on each level of respiratory support and $\mathrm{FiO}_{2}$ at 36 weeks' PMA (total $n=2169)$. Overall, $26.7 \%$ of infants were on $<1$ LPM $(n=$ 579); $13.4 \%$ were on $\geq 1$ to $\leq 1.5$ LPM $(n=291) ; 13.4 \%$ were on $>1.5$ to $\leq 2$ LPM $(n=290) ; 0.9 \%$ were on $>2$ to $<3$ LPM $(n=20) ; 13.9 \%$ were on $\geq 3$ LPM $(n=302) ; 5.9 \%$ were on NIMV $(n=128) ; 10.2 \%$ were on NCPAP $(n=222)$; and $15.5 \%$ were on CV/HFOV/HFJV $(n=$ 337). LPM liter per minute, NIMV nasal intermittent mechanical ventilation, NCPAP nasal continuous positive airway pressure, CV/ HFV conventional or high-frequency ventilation.

forms of BPD being discharged between 37 and 40 weeks' PMA, and those with severe BPD being discharged at a median of 43-48 weeks. Between $4 \%$ and $6 \%$ of infants with RS or Grade 1 BPD and $7-16 \%$ of infants with Grade 2 BPD, and $11-25 \%$ of infants with CNN BPD or Grade 3 BPD were being treated with bronchodilators, inhaled corticosteroids, or diuretics at discharge. The highest rates of tracheostomy were in the Grade 32019 NRN group, followed by the Grade 32018 NICHD group. Within the group of infants who had RS/ Grade 1-2 BPD, the 2019 NRN cohort had the highest rates of gastrostomy tube placement
(19\%), whereas infants classified by other definitions had rates between 6 and 10\%. Among infants with severe BPD, across all definitions, 20-36\% had gastrostomy tubes, and $5-7 \%$ had fundoplication during their hospitalization. The demographic and neonatal characteristics of the study cohort by BPD severity and classification are shown in Supplementary Table 2 and the characteristics of infants who died between 14 days and 36 weeks' PMA is shown in Supplementary Table 3.

\section{Multivariable models and relative risks}

To evaluate the effect of each severity level and definition on death, tracheostomy, and LOS, we developed multivariable regression models, adjusted for the center, gestational age, and sex (Table 2a). The effects of each grade in each definition on the outcomes were evaluated, and the common reference group allowed for direct comparisons of each BPD definition against the same baseline population (infants with no BPD by the 2019 NRN and CNN definitions). Severe groups in each classification discriminated those infants at the highest odds of death, and infants in the Grade 32019 NRN group had the highest odds. The odds of death within each classification increased in a step-wise fashion with increasing BPD severity, with the exception of infants in the Grade 12018 NICHD definition, who had a higher odds of death than those classified as having Grade 2 BPD. Infants who were in Grade 2 or 32019 NRN or 2018 NICHD groups were more likely to have a tracheostomy when compared with infants with no BPD, and LOS increased linearly with each increase in BPD severity level for all BPD classifications.

In order to understand the effect of $\mathrm{FiO}_{2}$ on relevant outcomes, we subdivided infants in the study cohort into categories of respiratory support and $\mathrm{FiO}_{2}$ and evaluated the relative risks of death and tracheostomy (Table $2 \mathrm{~b})$. Overall, 61 infants $(2.8 \%$ of 
Table 1. Short-term outcomes by classification.

\begin{tabular}{|c|c|c|c|c|c|}
\hline \multirow[t]{2}{*}{2019 NRN outcomes } & No BPD & Grade 1 & Grade 2 & Grade 3 & $p$-value \\
\hline & 1991 & 1161 & 672 & 337 & \\
\hline Died after 36 weeks PMA, $n$ (\%) & $2(0.1)$ & $5(0.4)$ & $11(1.6)$ & $43(12.8)$ & $<0.001$ \\
\hline Length of Stay (days from 36 weeks' PMA), median [IQR] & $12[5,25]$ & $28[15,43]$ & $50[35.5,76]$ & $84[51,130]$ & $<0.001$ \\
\hline Bronchodilators, $n(\%)$ & $6(0.3)$ & $71(6.1)$ & $107(16.0)$ & $81(24.0)$ & $<0.001$ \\
\hline Inhaled steroids, $n$ (\%) & $4(0.2)$ & $61(5.3)$ & $95(14.1)$ & $69(20.5)$ & $<0.001$ \\
\hline Tracheostomy, $n$ (\%) & $1(0.1)$ & - & $13(1.9)$ & $40(11.9)$ & $<0.001$ \\
\hline Gastrostomy, $n$ (\%) & $34(1.7)$ & $68(5.9)$ & $130(19.4)$ & $121(35.9)$ & $<0.001$ \\
\hline Fundoplication, $n(\%)$ & $7(0.4)$ & $15(1.3)$ & $36(5.4)$ & $21(6.2)$ & $<0.001$ \\
\hline \multirow[t]{2}{*}{2018 NICHD outcomes } & No BPD & Grade 1 & Grade 2 & Grade 3 & p-value \\
\hline & 2283 & 410 & 932 & 536 & \\
\hline Bronchodilators, $n$ (\%) & $19(0.8)$ & $35(8.5)$ & $78(8.4)$ & $133(24.8)$ & $<0.001$ \\
\hline Inhaled steroids, $n$ (\%) & $15(0.7)$ & $23(5.6)$ & $73(7.8)$ & $118(22.0)$ & $<0.001$ \\
\hline Diuretics, $n(\%)$ & $18(0.8)$ & $24(5.9)$ & 71 (7.6) & $85(15.9)$ & $<0.001$ \\
\hline Tracheostomy, $n$ (\%) & $1(0.0)$ & $1(0.2)$ & $8(0.9)$ & $44(8.2)$ & $<0.001$ \\
\hline Gastrostomy, $n(\%)$ & $53(2.3)$ & $41(10)$ & $97(10.4)$ & $162(30.2)$ & $<0.001$ \\
\hline Fundoplication, $n$ (\%) & $14(0.6)$ & $5(1.2)$ & $21(2.3)$ & $39(7.3)$ & $<0.001$ \\
\hline \multirow[t]{2}{*}{ CNN outcomes } & $\begin{array}{l}\text { CNN None/ } \\
\text { Mild }\end{array}$ & RS & & $B P D$ & $p$-value \\
\hline & 1991 & 871 & & 1229 & \\
\hline Died AFTER 36 weeks PMA, $n$ (\%) & $2(0.1)$ & $4(0.5)$ & & $55(4.2)$ & $<0.001$ \\
\hline Fundoplication, $n$ (\%) & $7(0.4)$ & $11(1.3)$ & & $61(4.7)$ & $<0.001$ \\
\hline
\end{tabular}

Short-term outcomes were evaluated within each BPD severity grade in the 2019 NRN, 2018 NICHD, and Canadian neonatal network (CNN) classifications. Chisquare or Fisher's Exact or Kruskal-Wallis tests were utilized to evaluated differences in proportions or medians across severity grades within one definition. Missing data were as follows: Mild or Grade 0, $n=9-12$; Grade 1, 2 or moderate, $n=11-24$; Grade 3 or severe, $n=21-89$.

infants on respiratory support) died between 36 weeks' PMA and hospital discharge, and 71 (3.3\%) received tracheostomies. The risk of death, relative to the risk for infants on room air, increased exponentially with increasing levels of respiratory support, particularly between $\leq 2$ LPM NC and non-invasive positive-pressure ventilation (>2 LPM, NIMV and NCPAP), and between non-invasive respiratory support and invasive respiratory support. Overall, mortality was infrequent in infants who were on the nasal cannula or non-invasive positive-pressure ventilation $(0.7 \%)$ and was highest for infants on mechanical ventilation (2\%). Tracheostomy followed a similar trend with $17(0.7 \%)$ on nasal cannula or non-invasive positive-pressure ventilation, and 53 out of 2169 (2.4\%) of infants on mechanical ventilation with this outcome. Because of the low frequency of death and tracheostomy in lower levels of respiratory support, the effect of the $\mathrm{FiO}_{2}$ category on outcomes could only be ascertained in infants on mechanical ventilation. Within this group, infants who were on $>0.7 \mathrm{FiO}_{2}$ at 36 weeks' PMA had a five-fold higher risk of death than those on $<0.3$.

\section{DISCUSSION}

In this study, we have compared short-term outcomes associated with three recent BPD definitions in a referral-based neonatal population. We found that infants with RS by the CNN definition or Grade 1 BPD by the 2019 NRN definition were unlikely to die or have severe complications, such as tracheostomy, despite being on the ventilator longer, requiring more postnatal steroids, and having more co-morbid conditions than infants without BPD. Infants with BPD by the CNN definition or Grade 2-3 BPD had a significantly increased odds of in-hospital mortality or tracheostomy, even when gestational age, gender, and center were controlled. The risk of death or tracheostomy increased 12-17fold for infants on $>2$ LPM NC relative to infants on no respiratory support, and those infants on NCPAP or NIMV at 36 weeks' PMA had an almost 50-fold increased risk of tracheostomy. As expected, the highest grade within any BPD definition was most associated with severe adverse outcomes, and infants in the Grade 32019 NRN definition had the highest odds of death or 
Table 2. Multivariable models by definition and relative risks by respiratory support level on outcomes. (a) Multivariable models of major outcomes by severity group and classification. (b) Frequency table and relative risk of respiratory support and $\mathrm{FiO}_{2}$ on death or tracheostomy.

\begin{tabular}{|c|c|c|c|c|c|c|}
\hline Classification & Death & $p$-value & Tracheostomy & $p$-value & LOS (days) & $p$-value \\
\hline Reference & & & & & $65(63,67)$ & \\
\hline \multicolumn{7}{|l|}{2019 NRN } \\
\hline Grade 1 & $6.1(1.1,33.3)$ & 0.04 & $1.5(0.1,24.8)$ & 0.78 & $77(75,80)$ & $<0.001$ \\
\hline Grade 2 & $20.7(4.3,99.3)$ & $<0.001$ & $56.2(7.1,444.1)$ & $<0.001$ & $96(92,99)$ & $<0.001$ \\
\hline Grade 3 & $225.0(50.1,>999)$ & $<0.001$ & $713.1(92.3,>999)$ & $<0.001$ & $120(114,125)$ & $<0.001$ \\
\hline \multicolumn{7}{|l|}{2018 NICHD } \\
\hline $\begin{array}{l}\text { No BPD } \\
(n=292)\end{array}$ & $4.0(0.4,45.3)$ & 0.26 & $8.1(0.5,131.9)$ & 0.14 & $81(78,85)$ & $<0.001$ \\
\hline Grade 1 & $21.6(4.3,107.9)$ & $<0.001$ & $10.5(0.9,119.1)$ & 0.06 & $83(79,86)$ & $<0.001$ \\
\hline Grade 2 & $6.8(1.2,40.0)$ & 0.03 & $22.8(2.7,188.6)$ & $<0.01$ & $82(79,85)$ & $<0.001$ \\
\hline Grade 3 & $145.2(32.4,651)$ & $<0.001$ & $351.9(45.9,>999)$ & $<0.001$ & $112(108,117)$ & $<0.001$ \\
\hline \multicolumn{7}{|l|}{ CNN } \\
\hline RS & $5.5(1.0,31.9)$ & 0.06 & - & - & $74(71,77)$ & $<0.001$ \\
\hline BPD & $45.9(10.6,199.0)$ & $<0.0001$ & $109.1(14.5,817.5)$ & $<0.001$ & $97(94,101)$ & $<0.001$ \\
\hline
\end{tabular}

(b) Frequency table and relative risk of respiratory support and $\mathrm{FiO}_{2}$ on death or tracheostomy

\section{Death}

\section{Respiratory support/FiO}

Room air (no support)

Nasal Cannula

$\begin{array}{ll}\leq 2 \text { LPM Any } \mathrm{FiO}_{2} & 1 \\ >2 \text { LPM Any } \mathrm{FiO}_{2} & 3 \\ \text { NIMV/NPCAP Any } \mathrm{FiO}_{2} & 350 \\ \text { CV/HFOV/HFJV } & 337 \\ <30 \% & 1 \\ \geq 30 \text { to } \leq 70 \% & 195 \\ >70 \% & 30\end{array}$

Totals

Tracheostomy

Respiratory support/FiO ${ }_{2}$

Room air (no support)

Nasal cannula

$\leq 2 \mathrm{LPM}$ any $\mathrm{FiO}_{2}$
$>2 \mathrm{LPM}$ any $\mathrm{FiO}_{2}$
NIMV/NPCAP
CV/HFOV/HFJV
$<30 \%$
$\geq 30$ to $\leq 70 \%$
$>70 \%$
Totals

\begin{tabular}{|c|c|c|}
\hline$N$ & Death, $n$ (\%) & RR Death $(95 \% \mathrm{Cl})$ \\
\hline 1991 & $2(0.1)$ & Ref \\
\hline $1160^{*}$ & $5(0.4)$ & $1.3(0.8,22.1)$ \\
\hline 322 & $5(1.6)$ & $15.5(3.0,79.3)$ \\
\hline 350 & $6(1.7)$ & $17.1(3.5,84.2)$ \\
\hline 337 & $43(12.8)$ & $127.0(30.9,521.9)$ \\
\hline 112 & $9(8.0)$ & Ref \\
\hline 195 & $21(10.8)$ & $1.3(0.6,2.8)$ \\
\hline 30 & $13(43.3)$ & $5.3(2.6,11.4)$ \\
\hline 4160 & $61(1.5)$ & \\
\hline$N$ & Tracheostomy, $n(\%)$ & RR Tracheostomy $(95 \% \mathrm{Cl})$ \\
\hline 1991 & $1(0.1)$ & Ref \\
\hline $1160^{\mathrm{a}}$ & $1(0.1)$ & $1.7(0.1,27.4)$ \\
\hline 322 & $2(0.6)$ & $12.4(1.1,136.0)$ \\
\hline 350 & $14(4.0)$ & $49.6(10.5,603.7)$ \\
\hline 337 & $53(15.7)$ & $313.1(43.4,2256.6)$ \\
\hline 112 & $18(16.1)$ & Ref \\
\hline 195 & $30(15.4)$ & $1.0(0.6,1.6)$ \\
\hline 30 & $5(16.7)$ & $1.0(0.4,2.6)$ \\
\hline 4160 & $71(1.7)$ & \\
\hline
\end{tabular}

Multivariable models, adjusted for Center, gestational age, and gender, were developed to evaluate the effect of each BPD grade within a definition on the outcomes of death and tracheostomy. The 2019 NRN Grade 0 and CNN "no BPD" infants $(n=1991)$ were a common reference group in logistic models. Generalized linear models were developed with median values for Center, gestational age, and gender $(F)$, serving as a reference. Shown are odds ratios and $95 \%$ confidence limits. $p$-values $<0.05$ are significant.

The number of deaths or tracheostomies were low in respiratory support levels of the nasal cannula and NIMV/NCPAP and could not be evaluated by FiO ${ }_{2}$ categories. The risks of death or tracheostomy in each respiratory support category relative to the risk for infants on no support ("room air") are shown in Table $2 \mathrm{~b}$. The oxygen categories under CV/HFOV/HFJV represent the risk of death or tracheostomy for infants on CV/HFOV/HFJV by FiO 2 level $(\geq 30$ to $\leq 70 \%$; $>70 \%$ ) relative to the risk for infants on mechanical ventilation and $<30 \% \mathrm{FiO}_{2}$. Relative risks and $95 \%$ confidence intervals are shown.

'One infant on Hood $\mathrm{O}_{2}$ at 36 weeks' PMA was removed from the analysis. 
tracheostomy, followed by the 2018 NICHD definition and then the CNN definition. These findings may allow clinicians to reassure families that infants on $\leq 2$ LPM NC at 36 weeks' PMA are unlikely to have severe in-hospital outcomes. However, these data support that infants in referral NICUs with intermediate grades of BPD have significantly increased risk for death and tracheostomy, and closemonitoring and therapeutic strategies targeted to preventing adverse outcomes in this population are needed. These data further corroborate the knowledge that infants on mechanical ventilation at 36 weeks' are at exponentially higher odds of death or tracheostomy and represent the most severe subset of infants with BPD.

Although the CNN RS and 2019 NRN Grade 1 definitions were similar and differed only in the threshold of nasal cannula flow, the infants classified as having BPD by the CNN definition ranged from being on relatively low levels of NC support to being on conventional ventilation. The heterogeneity of lung disease within the CNN definition seemed to diminish the associations between BPD and short-term outcomes in our cohort. In the publication by Isayama and colleagues, the BPD definition that was proposed was evaluated against long-term respiratory and neurodevelopmental outcomes, and as such, it may be less applicable to counseling regarding short-term outcomes [11]. Additionally, we found that infants categorized as having Grade 1 BPD by the 2018 NICHD definition had increased mortality compared to those who had Grade 2 BPD within that definition. A comparison of hospital characteristics between 2018 NICHD Grade 1-2 groups, and with the Grade 12019 NRN group, showed only a small increase in the proportion of infants who were growth-restricted (15\% vs $10 \%)$ in the Grade 12018 NICHD group. Although the etiology of the mortality increase is not clear in our cohort, possible explanations may lie in higher levels of respiratory support or factors other than BPD within the Grade 12018 NICHD group. Given these considerations with the CNN and 2018 NICHD definitions, we found that the 2019 NRN definition was the easiest to apply, and had the strongest discrimination for poor outcomes when utilized at 36 weeks' PMA.

The high morbidity and mortality seen for premature infants with the most severe forms of BPD at 36 weeks' PMA have been reported in other cohorts [14, 15]. In our cohort, the death and tracheostomy rates for each grade of BPD were slightly lower than those seen in the 2019 NRN cohort, potentially due to the longer length of follow-up in that investigation (18-26 months) [10]. However, compared with other studies of infants with severe BPD, more infants with Grade 3 BPD died in this study (8-13 vs 5\%) [16]. Although most neonates in our cohort were referred for prematurity without comorbid conditions (61\%), the second most common reason for referral was surgical evaluation (19\%) followed by respiratory diagnoses (10\%). Further, we included infants of greater gestational age ( $<32$ weeks) relative to the CNN and 2019 NRN cohorts in order to capture outcomes for a small but significant population of infants born between 29 and 31 weeks' who are referred to CHNC centers. However, all infants who had moderate or severe grades of BPD in our study were $\leq 28$ weeks' at birth, and more mature babies ( $>28$ weeks') fell into "No BPD" classifications. Due to the differences between the original cohort in which the CNN and 2019 NRN definition were derived, and the types of patients in referral-based neonatal intensive care units, it is important to validate the performance of newer BPD definitions in this population prior to adoption.

The largest numbers of infants in the cohort had low levels of respiratory support or Grade 1/2 BPD. By the CNN definition $21 \%$ of infants required RS, $32 \%$ had Grades 1 or 2 BPD by the 2018 NICHD definition, and $44 \%$ of infants had Grades 1 or 2 BPD by the 2019 NRN definition. In fact, nearly 54\% of infants who were on respiratory support at 36 weeks' PMA were on less than or equal to 2 LPM nasal cannula (Grade 1 BPD by the 2019 NRN definition). Additionally, we found that few infants were classified in certain respiratory support and $\mathrm{FiO}_{2}$ categories in our cohort. Only 20 infants were on $>2$ to $<3$ LPM of high-flow nasal cannula, 2 infants were on $>1$ LPM NC or non-invasive respiratory support and $\mathrm{FiO}_{2}$ $0.7-0.99$, and 13 infants were on $>1$ LPM NC or non-invasive respiratory support and $\mathrm{FiO}_{2}$ 1.0. The limited number of infants in these groups may reflect a clinical practice of increasing the level of respiratory support to avoid high levels of oxygen in infants with BPD. Non-invasive ventilatory strategies, particularly NCPAP and NIPPV, are commonly used as they prevent post-extubation failure, but they have not been shown to affect the incidence of BPD or mortality $[17,18]$. Further, although high-flow nasal cannula with higher rates of flow (3-8 LPM) may be used as an alternative to NCPAP, increasing durations of any form of noninvasive respiratory support beyond 60 days has been associated with increased death and neurodevelopmental impairment in very premature infants $[19,20]$. These findings support our caution that infants on non-invasive respiratory support are at risk for subsequent morbidity and mortality and warrant monitoring during hospitalization and post-discharge.

Although the role of $\mathrm{FiO}_{2}$ in defining BPD may be controversial, both animal models and human studies show that exposure to high oxygen concentration early in life may promote pathogenic lung remodeling and contribute to worse pulmonary outcomes in infancy [21-23]. Additional investigation regarding the value of including oxygen in BPD classifications at 36 weeks' PMA is warranted. We did find an increase in the relative risk of death or tracheostomy in a stepwise fashion as respiratory support level increased, but the number of events was limited and was not associated with $\mathrm{FiO}_{2}$ categories for infants on the nasal cannula or non-invasive respiratory support. For infants on mechanical ventilation, infants who were on higher $\mathrm{FiO}_{2}(>0.7)$ experienced death more often than those on lower $\mathrm{FiO}_{2}(<0.3)$, suggesting that $\mathrm{FiO}_{2}$ may have value in determining the short-term outcomes for infants within the most severe categories of BPD.

The LOS for our reference population was 65 days, similar to that seen by Ehrenkranz et al., although the LOS for the moderate and severe BPD categories for the 2001 definition was less in our cohort than in that cohort, possibly due to changes in clinical practice and discharge planning [24]. Although infants with No/ Mild BPD were discharged at a median PMA of 37 weeks', those with Grade 2 and 3 BPD were often discharged post-term, with 2019 NRN Grade 3 infants having a median PMA at the discharge of 48 weeks'. Given that even infants with moderate or Grade 1 BPD have a higher rate of respiratory medication use, gastrostomy and fundoplication, and LOS than do infants without BPD, classifications for BPD severity that are applied prior to 37 weeks' PMA are important for identifying at-risk populations prior to hospital discharge.

We acknowledge that this study has limitations. First, our centers do not universally utilize physiologic testing at 36 weeks' PMA, which may increase the numbers of infants on respiratory support or oxygen, and this may have contributed to the number of infants on low nasal cannula flow [25]. Additionally, our cohort may represent a sicker population overall as less than $4 \%$ of infants were inborn, with the remainder being transferred to level IV and referral-based units for care. This may have contributed to our rate of moderate/severe BPD using the 2001 NICHD definition being higher $(52 \%)$ than the $40-45 \%$ BPD rate reported in other large cohorts (data not shown) [26]. Finally, we recognize that the optimal timing of the application of the CNN definition may be after 36 weeks' PMA, but we chose a consistent time point for comparison and to increase applicability to clinicians, as many neonates are discharged before 40 weeks' PMA.

In conclusion, we have found that infants with Grade 3 BPD by the 2019 NRN classification system are at the highest risk of mortality and poor short-term outcomes, and we validated the utility of this definition in a referral-based neonatal population. Infants with Grade 2 BPD or with CNN BPD, particularly those on 
NCPAP or NIMV, are at higher risk for death, tracheostomy, respiratory medication use, and gastrostomy placement, and these infants, like those with Grade 3 BPD, should be monitored closely in the hospital and after discharge. The majority of infants who are on respiratory support at 36 weeks' PMA require $\leq 2$ LPM of nasal cannula flow, and as death and tracheostomy rates are low for this group, less severe markers of hospital outcomes should be considered for this population. Classification of BPD severity prior to 37 weeks' PMA is important so that multidisciplinary care and therapeutic intervention can occur in a timely manner prior to discharge for high-risk infants. Future efforts should focus on developing a unified classification system to support epidemiologic and clinical research and priority-setting for health services and research recommendations by national organizations and governing bodies.

\section{REFERENCES}

1. Northway WHJ, Rosan RC, Porter DY. Pulmonary disease following respirator therapy of hyaline-membrane disease-bronchopulmonary dysplasia. N Engl J Med. 1967;276:357-68.

2. Shennan AT, Dunn MS, Ohlsson A, Lennox K, Hoskins EM. Abnormal pulmonary outcomes in premature infants: prediction from oxygen requirement in the neonatal period. Pediatrics. 1988:82:527-32

3. Abman SH. Bronchopulmonary dysplasia: "a vascular hypothesis". Am J Respir Crit Care Med. 2001;164:1755-6.

4. Jobe AH, Bancalari E. Bronchopulmonary dysplasia. Am J Respir Crit Care Med. 2001;163:1723-9.

5. Poindexter BB, Feng R, Schmidt B, Aschner JL, Ballard RA, Hamvas A, et al. Comparisons and limitations of current definitions of bronchopulmonary dysplasia for the prematurity and respiratory outcomes program. Ann Am Thorac Soc. 2015;12:1822-30.

6. Ramaswamy VV, More K, Roehr CC, Bandiya P, Nangia S. Efficacy of noninvasive respiratory support modes for primary respiratory support in preterm neonates with respiratory distress syndrome: systematic review and network meta-analysis. Pediatr Pulmonol. 2020. https://doi.org/10.1002/ppul.25011.

7. Jain $D$, Bancalari E. New developments in respiratory support for preterm infants. Am J Perinatol. 2019;36:S13-S17.

8. Fernandez-Alvarez JR, Mahoney L, Gandhi R, Rabe H. Optiflow vs Vapotherm as extended weaning mode from nasal continuous positive airway pressure in preterm infants $\leq 28$ weeks gestational age. Pediatr Pulmonol. 2020. https://doi. org/10.1002/ppul.24936.

9. Higgins RD, Jobe AH, Koso-Thomas M, Bancalari E, Viscardi RM, Hartert TV, et al. Bronchopulmonary dysplasia: executive summary of a workshop. J Pediatr. 2018;197:300-8

10. Jensen EA, Dysart K, Gantz MG, McDonald S, Bamat NA, Keszler M et al. The diagnosis of bronchopulmonary dysplasia in very preterm infants: an evidencebased approach. Am J Respir Crit Care Med. 2019. https://doi.org/10.1164/ rccm.201812-23480C.

11. Isayama T, Lee SK, Yang J, Lee D, Daspal S, Dunn M, et al. Revisiting the definition of bronchopulmonary dysplasia: effect of changing panoply of respiratory support for preterm neonates. JAMA Pediatr. 2017;171:271-9.

12. Murthy K, Dykes FD, Padula MA, Pallotto EK, Reber KM, Durand DJ, et al. The Children's Hospitals Neonatal database: an overview of patient complexity, outcomes and variation in care. J Perinatol. 2014;34:582-6.

13. Olsen IE, Groveman SA, Lawson ML, Clark RH, Zemel BS. New intrauterine growth curves based on United States data. Pediatrics. 2010;125:e214-24.

14. McKinney RL, Schmidhoefer JJ, Balasco AL, Machan JT, Hirway P, Keszler M. Severe bronchopulmonary dysplasia: outcomes before and after the implementation of an inpatient multidisciplinary team. J Perinatol. 2020. https://doi. org/10.1038/s41372-020-00863-0.

15. Abman SH, Collaco JM, Shepherd EG, Keszler M, Cuevas-Guaman M, Welty SE, et al. Interdisciplinary care of children with severe bronchopulmonary dysplasia. J Pediatr. 2017;181:12-28.e1.

16. Jackson W, Hornik CP, Messina JA, Guglielmo K, Watwe A, Delancy G, et al. Inhospital outcomes of premature infants with severe bronchopulmonary dysplasia. J Perinatol. 2017:37:853-6.
17. Ekhaguere $\mathrm{O}$, Patel $\mathrm{S}$, Kirpalani $\mathrm{H}$. Nasal intermittent mandatory ventilation versus nasal continuous positive airway pressure before and after invasive ventilatory support. Clin Perinatol. 2019;46:517-36.

18. Lemyre B, Davis PG, De Paoli AG, Kirpalani H. Nasal intermittent positive pressure ventilation (NIPPV) versus nasal continuous positive airway pressure (NCPAP) for preterm neonates after extubation. Cochrane Database Syst Rev. 2017;2: CD003212.

19. Cummings JJ, Polin RA. Noninvasive respiratory support. Pediatrics. 2016; 137. https://doi.org/10.1542/peds.2015-3758.

20. Zhang H, Dysart K, Kendrick DE, Li L, Das A, Hintz SR, et al. Prolonged respiratory support of any type impacts outcomes of extremely low birth weight infants. Pediatr Pulmonol. 2018;53:1447-55.

21. Dylag AM, Kopin HG, O'Reilly MA, Wang $H$, Davis SD, Ren $C L$, et al. Early neonatal oxygen exposure predicts pulmonary morbidity and functional deficits at 1 year. J Pediatr. 2020;223:20-28.e2.

22. Ha AW, Sudhadevi T, Ebenezer DL, Fu P, Berdyshev EV, Ackerman SJ, et al. Neonatal therapy with PF543, a sphingosine kinase 1 inhibitor, ameliorates hyperoxia-induced airway remodeling in a murine model of bronchopulmonary dysplasia. Am J Physiol Lung Cell Mol Physiol. 2020;319:L497-L512.

23. Chen S, Wu Q, Zhong D, Li C, Du L. Caffeine prevents hyperoxia-induced lung injury in neonatal mice through NLRP3 inflammasome and NF-KB pathway. Respir Res. 2020;21:140.

24. Ehrenkranz RA, Walsh MC, Vohr BR, Jobe AH, Wright LL, Fanaroff AA, et al. Validation of the National Institutes of Health consensus definition of bronchopulmonary dysplasia. Pediatrics. 2005;116:1353-60.

25. Walsh MC, Yao Q, Gettner P, Hale E, Collins M, Hensman A, et al. Impact of a physiologic definition on bronchopulmonary dysplasia rates. Pediatrics. 2004;114:1305-11.

26. Stoll BJ, Hansen NI, Bell EF, Walsh MC, Carlo WA, Shankaran S, et al. Trends in care practices, morbidity, and mortality of extremely preterm neonates, 1993-2012. JAMA. 2015:314:1039-51.

\section{AUTHOR CONTRIBUTIONS}

Author contributions to this manuscript are as follows: study design, data analysis, and preparation of the manuscript (SVR, JML); data analysis and manuscript review (JWL, ACC, JM, KTL, RSR, MM, EW, JWI, RJD, SY, BEH, MAP, NFP, KM, LDN, CHC, RCS, WET, and WAE); Statistical analyses and manuscript review (IZ).

\section{FUNDING}

The Children's Hospitals Neonatal Consortium (501-c3 organization) supported the statistical analyses presented in this manuscript.

\section{COMPETING INTERESTS}

The authors declare no competing interests.

\section{ETHICS APPROVAL AND CONSENT TO PARTICIPATE}

De-identified data analyses were approved by the Institutional Review Board of Stanley Manne Research Institute affiliated with the Ann and Robert H. Lurie Children's Hospital of Chicago. All participating centers obtained regulatory oversight to participate in the CHNC.

\section{ADDITIONAL INFORMATION}

Supplementary information The online version contains supplementary material available at https://doi.org/10.1038/s41372-021-01178-4.

Correspondence and requests for materials should be addressed to S.V-R.

Reprints and permission information is available at http://www.nature.com/ reprints

Publisher's note Springer Nature remains neutral with regard to jurisdictional claims in published maps and institutional affiliations. 